\section{The Turing mechanism in vertebrate limb patterning}

\section{Stuart A. Newman}

In the Review 'Making digit patterns in the vertebrate limb', Cheryll Tickle discusses evidence that the long-standing concept of 'positional information' - which was formulated to account for differences in the appearance of distinct skeletal elements - is inadequate to explain the number and arrangement of such elements. As an alternative means for laying out the overall template for the limb skeleton, she briefly mentions an entirely different mechanism of pattern formation: the reaction-diffusion model, which was originally put forward by the mathematician A.M. Turing ${ }^{2}$. As Tickle states, "The series of many unpatterned digits, which develop in the absence of GLI3 function in mouse mutants, is reminiscent of the digit pre-pattern that has been proposed to function in combination with the morphogen gradient ${ }^{[3]}$. According to this proposal, a series of digit condensations, a pre-pattern, is specified by a wave-like distribution of a morphogen that is generated by a reaction-diffusion mechanism, with the peaks corresponding to the condensations. A gradient of another morphogen ... then provides each peak with a positional value and a digit identity. The number of peaks that are generated by the reaction-diffusion mechanism depends on the width of the limb."

The proposal that the limb skeleton is generated by a reaction-diffusion mechanism, with other morphogen gradients having a fine-tuning role, was first made not in the 1989 review $^{3}$ that she cites, but a decade previously, in conjunction with a specific model for the production of patterns of pre-cartilage condensation through the interaction of mesenchymal cells with morphogens and with the extracellular matrix $(\mathrm{ECM})^{4}$. More importantly, Tickle failed to describe research in the ensuing three decades that has seriously considered, tested and elaborated on the role of reactiondiffusion patterning in limb development. This includes a series of papers by Takashi Miura and his colleagues that provided evidence for a reaction-diffusion mechanism over a mechanochemical alternative in an in vitro model for pre-chondrogenic pattern formation ${ }^{5}$, and for the functionality of transforming growth factor- $\beta 2$ (TGF $\beta 2$ ) as the activator in a reaction-diffusion scheme $^{6}$. Later, it was demonstrated that lateral inhibition of limb pre-cartilage condensation (a necessary component of the reaction-diffusion framework) was induced by ectodermally produced fibroblast growth factors $(\mathrm{FGFs})^{7}$. This was followed by a reaction-diffusion-based mathematical analysis ${ }^{8}$ that accounted for the coordinate effects of FGFs in laterally inhibiting and accelerating the rate of appearance of foci of pre-cartilage condensation. Miura and co-workers also showed that the peculiar 'thick-thin' morphology of limb skeletal elements that is observed in doublefoot-mutant mice could be understood by assuming that a reaction-diffusion mechanism underlies skeletal patterning?.

In the original reaction-diffusion-based limb model, the hypothesis that the nondiffusible ECM protein fibronectin mediates pre-cartilage condensation as part of a regulatory circuit that involves diffusible morphogens $s^{4}$ has been confirmed in several experimental studies (reviewed in REF. 10). Recently, the functionality of fibronectin has been incorporated into a 'reactor-diffusion' mode ${ }^{11}$ in which authentic cell behaviours are taken into account. The reactor-diffusion approach has enabled the introduction of increasing levels of biological detail, mathematical rigour and computational sophistication into models for chondrogenic pattern formation in vivo and in vitro ${ }^{12-15}$.

Far from being a sideline in studies of vertebrate limb development, experimentally motivated reaction-diffusion and reactordiffusion models, along with mathematically related models based on biochemicalgenetic oscillations, have gained prominence in many areas of developmental biology $y^{10,16}$, including patterning of the hair follicles ${ }^{17}$, feather germs ${ }^{18}$ and teeth ${ }^{19}$ and, in the oscillatory mode, somites ${ }^{20}$. Indeed, with regard to the generation of patterns with repeating elements, this class of dynamic mechanisms has eclipsed models that are based primarily on positional information.

Department of Cell Biology and Anatomy, Basic Science Building, New York Medical College, Valhalla, New York 10595, USA. e-mail:newman@nymc.edu doi:10.1038/nrm1830-c1

Tickle, C. Making digit patterns in Nature Rev. Mol. Cell Biol. 7, 45-53 (2006). Turing, A. The chemical basis of morphogenesis. Phil. Trans. R. Soc. Lond. B 237, 37-72 (1952). Wolpert, L. Positional information revisited. Development 107, 3-12 (1989).

4 Newman, S. A. \& Frisch, H. L. Dynamics of skeletal pattern formation in developing chick limb. Science 205, 662-668 (1979)

5. Miura, T. \& Shiota, K. Extracellular matrix Miura, T. \& Shiota, K. Extracellular matrix
environment influences chondrogenic pattern formation in limb bud micromass culture: experimental verification of theoretical models. Anat. Rec. 258, 100-107 (2000).

6. Miura, T. \& Shiota, K. TCF $\beta 2$ acts as an 'activator' molecule in reaction-diffusion model and is involved in cell sorting phenomenon in mouse limb micromass culture. Dev. Dyn. 217, 241-249 (2000).

7. Moftah, M. Z. et al. Ectodermal FGFs induce perinodular inhibition of limb chondrogenesis in vitro and in vivo via FGF receptor 2. Dev. Biol. 249 270-282 (2002).

8. Miura, T. \& Maini, P. K. Speed of pattern appearance in reaction-diffusion models: implications in the pattern formation of limb bud mesenchyme cells Bull. Math. Biol. 66, 627-649 (2004).

9. Miura, T., Shiota, K., Morriss-Kay, G. \& Maini, P. K. Mixed-mode pattern in doublefoot mutant mouse limb - Turing reaction-diffusion model on a growing domain during limb development. J. Theor. Biol. 240 , 562-573 (2006).

10. Forgacs, G. \& Newman, S. A. Biological Physics of the Developing Embryo (Cambridge Univ. Press, Cambridge, 2005)

11. Hentschel, H. G., Glimm, T., Glazier, J. A. \& Newman, S. A. Dynamical mechanisms for skeletal pattern formation in the vertebrate limb. Proc. R. Soc Lond. B. 271, 1713-1722 (2004).

12. Chaturvedi, R. et al. On multiscale approaches to three-dimensional modelling of morphogenesis. J. R. Soc. Interface 2, 237-253 (2005).

13. Cickovski, T. et al. A framework for three-dimensional simulation of morphogenesis. IEEE/ACM Trans. Comput. Biol. Bioinform. 2, 273-288 (2005).

14. Christley, S., Alber, M. S. and Newman, S. A. Patterns of mesenchymal condensation in a multiscale, discrete stochastic model. PLoS Comput. Biol. 3, e76 (2007).

15. Alber, M., Hentschel, H. G. E., Kazmierczak, B. \& Newman, S. A. Existence of solutions to a new model of biological pattern formation. J. Math. Anal. Appl. 308, 175-194 (2005)

16. Maini, P. K., Baker, R. E. \& Chuong, C. M. Developmental biology. The Turing model comes of molecular age. Science 314, 1397-1398 (2006).

17. Sick, S., Reinker, S., Timmer, J. \& Schlake, T. WNT and DKK determine hair follicle spacing through a reaction-diffusion mechanism. Science 314 reaction-diffusion m)

18. Jiang, T. X. et al. Integument pattern formation involves genetic and epigenetic controls: feather arrays simulated by digital hormone models. Int. J. Dev. Biol. 48, 117-135 (2004).

19. Salazar-Ciudad, I. \& Jernvall, J. A gene network mode accounting for development and evolution of mammalian teeth. Proc. Natl Acad. Sci. USA 99 8116-8120 (2002).

20. Baker, R. E., Schnell, S. \& Maini, P. K. A clock and wavefront mechanism for somite formation. Dev. Biol. 293, 116-126 (2006). 